\title{
Coupling-Independent Capacitive Wireless Power Transfer Using Frequency Bifurcation
}

\author{
Ben Minnaert ${ }^{1, *(\mathbb{D})}$, Franco Mastri $\left.{ }^{2} \mathbb{(}\right)$, Nobby Stevens ${ }^{1}$, Alessandra Costanzo ${ }^{2}$ \\ and Mauro Mongiardo ${ }^{3}$ \\ 1 KU Leuven, DRAMCO, Department of Electrical Engineering (ESAT), Technology Campus Ghent, \\ 9000 Ghent, Belgium; nobby.stevens@kuleuven.be \\ 2 Department of Electrical, Electronic and Information Engineering Guglielmo Marconi, \\ University of Bologna, 40136 Bologna, Italy; franco.mastri@unibo.it (F.M.); \\ alessandra.costanzo@unibo.it (A.C.) \\ 3 Department of Engineering, University of Perugia, 06123 Perugia, Italy; mauro.mongiardo@unipg.it \\ * Correspondence: ben.minnaert@kuleuven.be; Tel.: +32-9-331-6547
}

Received: 28 June 2018; Accepted: 20 July 2018; Published: 22 July 2018

\begin{abstract}
Capacitive wireless power transfer can be realized by mutually coupled capacitors operating at a common resonant frequency. An optimal load exists that maximizes either the efficiency or the power transfer to the load. In this work, we utilize the frequency bifurcation effect to propose a frequency agile mode that allows for a nearly coupling-independent regime. We analytically determine the operating conditions of the coupling-independent mode based on the different system gains. In this way, we obtain a solution that achieves nearly constant efficiency and power transfer, even at varying coupling. We compare our results to inductive wireless power transfer where a perfect coupling-independent mode is achievable.
\end{abstract}

Keywords: capacitive wireless power transfer; coupling factor; frequency bifurcation; resonance; wireless power transfer

\section{Introduction}

Wireless power transfer (WPT) allows energy transfer from a transmitter to a receiver across an air gap, without any electrical connections [1,2]. Technically, any device that needs power can become an application for WPT. The current list of applications where WPT is applied, is therefore very diverse [3,4]. We limit ourselves to some examples. Most known WPT applications are portable electronics and household devices (e.g., smartphones, electric toothbrushes, wearables, smart watches). WPT is especially important for biomedical implants such as pacemakers [5], neurostimulators [6] and spinal cord stimulators [7].

The above examples are located in the low to midrange power levels, from a few watt to $100 \mathrm{~W}$. However, WPT also allows for high power transfer, well above kilowatt level (at short distance) and is therefore used by industrial automation (e.g., automated guided vehicles [8], robots [9], and automated underwater vehicles [10]). An important issue hindering the breakthrough of electric vehicles is the limited action radius and the hassle to frequently connect the power cable. Wireless charging could improve the adaptation to electric vehicles significantly by providing wireless charging points at parking lots, in the garage, or at bus stops for electric public transport [11,12]. Even wireless charging while driving is being pursued by installing transmitters in motorway lanes $[13,14]$.

Other promising markets for WPT include the roll-out of Internet of Things (IoT) sensor and communication networks [15,16], machine-to-machine communication systems [17,18], powering unmanned aerial vehicles [19], and on-body sensor networks for animals [20]. 
A promising WPT technique that has already entered as well the consumer as the industrial market is inductive wireless power transfer (IPT) [2,21-23]. This near-field technique uses a varying magnetic field to transfer energy from a transmitter coil to a coupled receiver coil. Another near-field technique is capacitive wireless power transfer (CPT) that uses the electric field to transfer energy from a transmitter plate to a receiver plate. However, CPT has not yet significantly entered the market and its technology is not as maturely developed as IPT. Compared to IPT, CPT has the advantage to transfer energy through metal, and is expected to realize WPT at a lower cost and weight than IPT [22,24].

In general, a practical WPT system design will pursue one of two options, depending on the application $[2,25,26]$. It will either

- optimize the efficiency of the system, e.g., for the wireless charging of high power applications as electric vehicles.

- $\quad$ optimize the power transfer to the load, e.g., charging transcutaneous biomedical implants.

Two approaches exist to achieve these solutions [27]:

- In a fixed frequency design, an impedance compensation network is added to realize optimum efficiency or power transfer at the operating frequency. The value of its components are independent of the coupling between transmitter and receiver, but for each different value of the coupling, another optimal load value applies. This approach has the advantage of realizing optimum efficiency or power transfer at a fixed operating frequency. However, at fluctuating coupling, the load value has to change to retain the optimal conditions.

- In a frequency agile design, optimum efficiency or power transfer can be achieved for a fixed load value, even at fluctuating coupling. With the same impedance compensation network as in the fixed frequency design, a constant efficiency or power transfer can be realized by changing the operating frequency, depending on the coupling between transmitter and receiver.

For applications where the coupling is variable in time, the latter scheme is preferred.

Recently, an analytical solution was given for realizing the frequency agile design by applying frequency bifurcation for inductive wireless power transfer [27-29]. It allows for a coupling-independent IPT system by sweeping the frequency to realize a purely resistive input impedance.

However, to our knowledge, no analytic coupling-independent solution was presented yet for CPT. In this work, we study the fundamental design for achieving a CPT link that is independent of the coupling between transmitter and receiver by using a frequency agile approach. More specifically, our contributions are as follows:

- $\quad$ determination of the power and transducer gain for a general CPT system;

- analytical calculation of the bifurcation conditions and frequencies, necessary to determine the optimal operating frequency;

- analytical computation of the optimal solution for achieving a practically coupling-independent CPT link;

- $\quad$ illustration of the similarities to IPT.

\section{Methodology}

We consider a general, static CPT system. We focus on the wireless link itself and neglect any remote electronics such as drivers and power conditioner. The capacitive link can be represented by an equivalent circuit, given by capacitances $C_{1}$ and $C_{2}$, coupled by their mutual capacitance $C_{M}$ (Figure 1 ). We refer to [30] for the measurement procedure to determine the value of these capacitances for a general CPT set-up. The coupling factor $k$ is given by:

$$
k=\frac{C_{M}}{\sqrt{C_{1} C_{2}}}
$$


Based on Norton's theorem, we represent the supply of the transmitter by a time-harmonic current source $I_{S}$ (peak value) with shunt conductance $G_{S}$. Power is wireless transmitted to the load conductance $G_{L}$. We create resonant circuits by adding the shunt inductances $L_{1}$ and $L_{2}$ to the transmitter and receiver, respectively, given by $(i=1,2)$ :

$$
L_{i}=\frac{1}{\omega_{0}^{2} C_{i}}
$$

with $\omega_{0}$ the chosen resonant angular frequency. The inductor and capacitor losses are represented by the series resistances $R_{L 1}, R_{L 2}, R_{C 1}$ and $R_{C 2}$ as indicated on Figure 1 . It should be noted that the introduction of these resistances slightly modifies the resonant frequencies of the two resonators, which become:

$$
\omega_{0 i}^{\prime}=\omega_{0} \sqrt{\frac{Q_{C i}^{2}}{Q_{L i}^{2}} \frac{1-Q_{L i}^{2}}{1-Q_{C i}^{2}}}
$$

where $Q_{L i}=\omega_{0} L_{i} / R_{L i}$ and $Q_{C i}=1 / \omega_{0} R_{C i} C_{i}$ are the inductor and capacitor quality factors, respectively $(i=1,2)$. In practical cases this variation is very small and can be neglected. Anyhow, it can be observed that for different transmitter and receiver quality factors, the resonators are not synchronous, i.e., they have different resonant frequencies. In the following it will be shown that this may affect the coupled resonators behavior.



Figure 1. Equivalent circuit representation of a general capacitive wireless power transfer (CPT) system, indicating the input admittance $Y_{i n}$, output admittance $Y_{\text {out }}$, and input power $P_{\text {in }}$.

Figure 1 shows the equivalent circuit for the general CPT system, indicating the definition for the input admittance $Y_{i n}=G_{i n}+j B_{\text {in }}$ and output admittance $Y_{\text {out }}=G_{\text {out }}+j B_{\text {out }}$. We consider the wireless link as a two-port network, characterized by its admittance matrix $Y$ (Figure 1):

$$
Y=\left[\begin{array}{cc}
\frac{1}{R_{L 1}+j \omega L_{1}}+\frac{1}{R_{C 1}+\frac{1}{j \omega C_{1}}} & -j \omega C_{M} \\
-j \omega C_{M} & \frac{1}{R_{L 2}+j \omega L_{2}}+\frac{1}{R_{C 2}+\frac{1}{j \omega C_{2}}}
\end{array}\right]
$$

To generalize our analysis, we perform the following normalizations. We define the normalized frequency $u$ as:

$$
u=\frac{\omega}{\omega_{0}}
$$

We normalize the different quantities as given by Table 1 . Notice that $r_{L i}$ and $r_{C i}$ are the reciprocal of the quality factors $Q_{L i}$ and $Q_{C i}(i=1,2)$, respectively. Analogously, $g_{S}$ and $g_{L}$ correspond with the reciprocal of the external quality factors. The admittance matrix $Y$ can then be written as:

$$
\boldsymbol{Y}=\left[\begin{array}{cc}
\omega_{0} C_{1}\left[\frac{r_{C 1} u^{2}}{1+r_{C 1}^{2} u^{2}}+\frac{r_{L 1}}{r_{L 1}^{2}+u^{2}}+j\left(\frac{u}{1+r_{C 1}^{2} u^{2}}-\frac{u}{r_{L 1}^{2}+u^{2}}\right)\right] & -j \sqrt{C_{1} C_{2}} \omega_{0} k u \\
-j \sqrt{C_{1} C_{2}} \omega_{0} k u & \omega_{0} C_{2}\left[\frac{r_{C 2} u^{2}}{1+r_{C 2}^{2} u^{2}}+\frac{r_{L 2}}{r_{L 2}^{2}+u^{2}}+j\left(\frac{u}{1+r_{C 2}^{2} u^{2}}-\frac{u}{r_{L 2}^{2}+u^{2}}\right)\right]
\end{array}\right]
$$


Table 1. Normalized quantities for CPT.

\begin{tabular}{ll}
\hline$g_{S}=G_{S} /\left(\omega_{0} C_{1}\right)=1 / Q_{S}$ & $g_{L}=G_{L} /\left(\omega_{0} C_{2}\right)=1 / Q_{L}$ \\
$r_{L 1}=R_{L 1} /\left(\omega_{0} L_{1}\right)=1 / Q_{L 1}$ & $r_{L 2}=R_{L 2} /\left(\omega_{0} L_{2}\right)=1 / Q_{L 2}$ \\
$r_{C 1}=\omega_{0} C_{1} R_{C 1}=1 / Q_{C 1}$ & $r_{C 2}=\omega_{0} C_{2} R_{C 2}=1 / Q_{C 2}$ \\
$g_{\text {in }}=G_{\text {in }} /\left(\omega_{0} C_{1}\right)$ & $g_{\text {out }}=G_{\text {out }} /\left(\omega_{0} C_{2}\right)$ \\
$b_{\text {in }}=B_{\text {in }} /\left(\omega_{0} C_{1}\right)$ & $b_{\text {out }}=B_{\text {out }} /\left(\omega_{0} C_{2}\right)$ \\
\hline
\end{tabular}

If the quality factors are high, it is possible to simplify the admittance matrix by neglecting the normalized resistances with respect to $u^{2}$. We obtain the approximate admittance matrix $Y_{a}$ given by:

$$
Y_{a}=\left[\begin{array}{cc}
\omega_{0} C_{1}\left[r_{C 1} u^{2}+\frac{r_{L 1}}{u^{2}}+j \frac{u^{2}-1}{u}\right] & -j \sqrt{C_{1} C_{2}} \omega_{0} k u \\
-j \sqrt{C_{1} C_{2}} \omega_{0} k u & \omega_{0} C_{2}\left[r_{C 2} u^{2}+\frac{r_{L 2}}{u^{2}}+j \frac{u^{2}-1}{u}\right]
\end{array}\right]
$$

For ease of notation, we introduce the following definitions:

$$
\begin{gathered}
a=1+2 g_{L} r_{C 2} \\
b=2-g_{L}^{2} \\
c=1+2 g_{L} r_{L 2} \\
d=r_{L 1}+r_{L 2} \\
e=r_{C 1}+r_{C 2} \\
r_{1}=r_{L 1}+r_{C 1} \\
r_{2}=r_{L 2}+r_{C 2}
\end{gathered}
$$

The input admittance $Y_{i n}$ is determined in the Appendix A. We obtain for the normalized input admittance $y_{i n}=g_{\text {in }}+j b_{\text {in }}$ (neglecting $r_{L 2}^{2}, r_{C 2}^{2}$ and $r_{L 2} r_{C 2}$ with respect to $u^{2}$ ):

$$
\begin{gathered}
g_{\text {in }}=r_{C 1} u^{2}+\frac{r_{L 1}}{u^{2}}+\frac{k^{2} u^{2}\left(r_{C 2} u^{4}+g_{L} u^{2}+r_{L 2}\right)}{a u^{4}-b u^{2}+c} \\
b_{i n}=\frac{\left(u^{2}-1\right)}{u} \frac{\left(a-k^{2}\right) u^{4}-b u^{2}+c}{a u^{4}-b u^{2}+c}
\end{gathered}
$$

The imaginary part of the input admittance, $b_{i n}$, is zero if the normalized frequency $u$ equals 1 , i.e., at the resonant angular frequency $\omega_{0}$. This frequency is called the main resonant frequency. However, Equation (16) demonstrates that other frequencies exist where the imaginary part of the input admittance is zero. Indeed, if we pose $x=u^{2}$, the other frequencies are found by solving the quadratic equation

$$
\left(a-k^{2}\right) x^{2}-b x+c=0
$$

Based on Descartes' sign rule and the requirement of a nonnegative discriminant of the quadratic equation (with $0 \leq k \leq 1$ ), we find the conditions for which these other frequencies exist:

$$
\begin{gathered}
g_{L} \leq \sqrt{2} \\
k \geq k_{B}=\sqrt{a-\frac{b^{2}}{4 c}}
\end{gathered}
$$

$k_{B}$ is called the bifurcation coupling. If the two above conditions apply, two other frequencies besides the main resonant frequency exist where $b_{i n}$ equals zero. These frequencies are called the secondary resonant frequencies. Solving Equation (17), we find: 


$$
u_{ \pm}=\sqrt{\frac{b \pm \sqrt{b^{2}-4 c\left(a-k^{2}\right)}}{2\left(a-k^{2}\right)}}
$$

At the main $(u=1)$ and secondary $\left(u=u_{ \pm}\right)$resonant frequencies, the normalized input admittance is real and equals

$$
\begin{gathered}
g_{\text {in }}(u=1)=r_{1}+\frac{k^{2}}{2\left(g_{L}+2 r_{2}\right)}+\frac{k^{2}}{2 g_{L}} \\
g_{\text {in }}\left(u=u_{ \pm}\right)=g_{L}+\frac{b d}{c}+\frac{1}{2}\left(\frac{e}{a-k^{2}}-\frac{d}{c}\right)\left[b \pm \sqrt{b^{2}-4 c\left(a-k^{2}\right)}\right]
\end{gathered}
$$

To optimize a WPT network for either efficiency or power transfer, two power gains are of interest [31]:

- The power gain $G_{P}$, defined as the ratio between the power $P_{L}$ dissipated by the load and the input power $P_{i n}$ of the network. This definition corresponds with the efficiency definition often applied in the context of WPT [32]. Maximizing $G_{P}$ corresponds with maximizing the efficiency of the system.

- The transducer gain $G_{T}$ is defined as the ratio between the power $P_{L}$ dissipated by the load and the maximum available power $P_{A G}$ of the generator. For a fixed $P_{A G}$, maximizing $G_{T}$ corresponds to maximizing the amount of power transferred to the load.

Both gain expressions as function of the admittance matrix of a two-port network are determined in the Appendix A.

For the admittance matrix (7), we obtain after a simple but elaborate algebraic restatement, and neglecting $r_{L 2}^{2}, r_{C 2}^{2}$ and $r_{L 2} r_{C 2}$ with respect to $u^{2}$ :

$$
\begin{gathered}
G_{P}=\frac{k^{2} u^{4}}{a u^{4}-b u^{2}+c} \frac{g_{L}}{g_{i n}} \\
G_{T}=\frac{4 g_{\text {in }} g_{S}}{\left|y_{\text {in }}+g_{S}\right|^{2}} G_{P}
\end{gathered}
$$

As is to be expected, the power gain $G_{P}$ depends on the load $g_{L}$, but not on the source conductance $g_{S}$. The transducer gain $G_{T}$ is dependent on both $g_{S}$ and $g_{L}$.

At the main resonant frequency $(u=1)$ and the secondary $\left(u=u_{ \pm}\right)$resonant frequencies, the gains are given by:

$$
\begin{gathered}
G_{P}(u=1)=\frac{k^{2} g_{L}}{g_{L} r_{1}\left(g_{L}+2 r_{2}\right)+k^{2}\left(g_{L}+r_{2}\right)} \\
G_{P}\left(u=u_{ \pm}\right)=\frac{g_{L}}{g_{L}+\frac{b d}{c}+\frac{1}{2}\left(\frac{e}{a-k^{2}}-\frac{d}{c}\right)\left[b \pm \sqrt{b^{2}-\sqrt{b^{2}-4 c\left(a-k^{2}\right)}}\right]} \\
G_{T}(u=1)=\frac{4 k^{2} g_{S} g_{L}^{2}\left(g_{L}+2 r_{2}\right)}{\left[k^{2}\left(g_{L}+r_{2}\right)+g_{L}\left(g_{S}+r_{1}\right)\left(g_{L}+2 r_{2}\right)\right]^{2}} \\
G_{T}\left(u=u_{ \pm}\right)=\frac{4 g_{S} g_{L}}{\left[g_{S}+g_{L}+\frac{b d}{c}+\frac{1}{2}\left(\frac{e}{a-k^{2}}-\frac{d}{c}\right)\left[b \pm \sqrt{b^{2}-4 c\left(a-k^{2}\right)}\right]\right]^{2}}
\end{gathered}
$$

It can be noted that in the lossles case $\left(r_{C i}=0, r_{L i}=0\right)$, the denominators of Equations (26) and (28) reduce to $g_{L}$ and $\left(g_{S}+g_{L}\right)^{2}$, respectively. Hence, at the secondary resonances, the gains are independent of $k$. On the other hand, if the inductor and capacitor quality factors are high, the coupling-dependent terms in Equations (26) and (28) are generally small with respect to $g_{L}$ and, consequently, nearly coupling-independent gains can be achieved. 
We have determined analytical expressions for the secondary resonant frequencies and the corresponding gains under the assumption that the quality factors are high, i.e., for the admittance matrix $Y_{a}$. If we apply the same methodology for the general admittance matrix $Y$, the numerator of Equation (16) results in a quartic equation in $u^{2}$ which cannot be solved analytically, unless in the particular case when both inductors and capacitors have the same quality factor (i.e., if both resonator circuits are synchronous).

For asynchronous resonators, an analytical solution is not available. Depending on $k, 2$ or 4 solutions can be found. Besides the main and secondary resonant frequencies, another resonance is found, valid for the entire range of $k$, which is not present for the approximated model. However, this fourth resonance is very large for practical values of $k(u>100)$, and can therefore be neglected.

In the next section, we will not only discuss the derived analytical model (based on the approximated admittance matrix $\boldsymbol{Y}_{a}$ ), but also the exact model (based on $\boldsymbol{Y}$ ) by numerical computation. We will demonstrate the validity of applying the approximated analytical model for WPT applications.

\section{Discussion}

We discuss the results by considering an asynchronous CPT system with $g_{S}=r_{L 1}=0.01, r_{L 2}=0.015$, $r_{C 1}=r_{C 2}=0.005$ and a load of $g_{L}=0.15$. This corresponds with quality factors $Q_{L 1}=100, Q_{L 2}=66.7$ and $Q_{C 1}=Q_{C 2}=200$. Figure 2 shows the normalized frequency as function of the coupling coefficient $k$ for this configuration. Frequency bifurcation occurs for a coupling factor $k$ higher than the bifurcation coupling $k_{B}=16.8 \%$. The solid lines indicate the results for the approximated model, based on admittance matrix $Y_{a}$. We notice a perfect pitchfork bifurcation. The dashed lines show the result for the exact model, based on admittance matrix $Y$. We observe an imperfect pitchfork bifurcation: two separate branches are present.

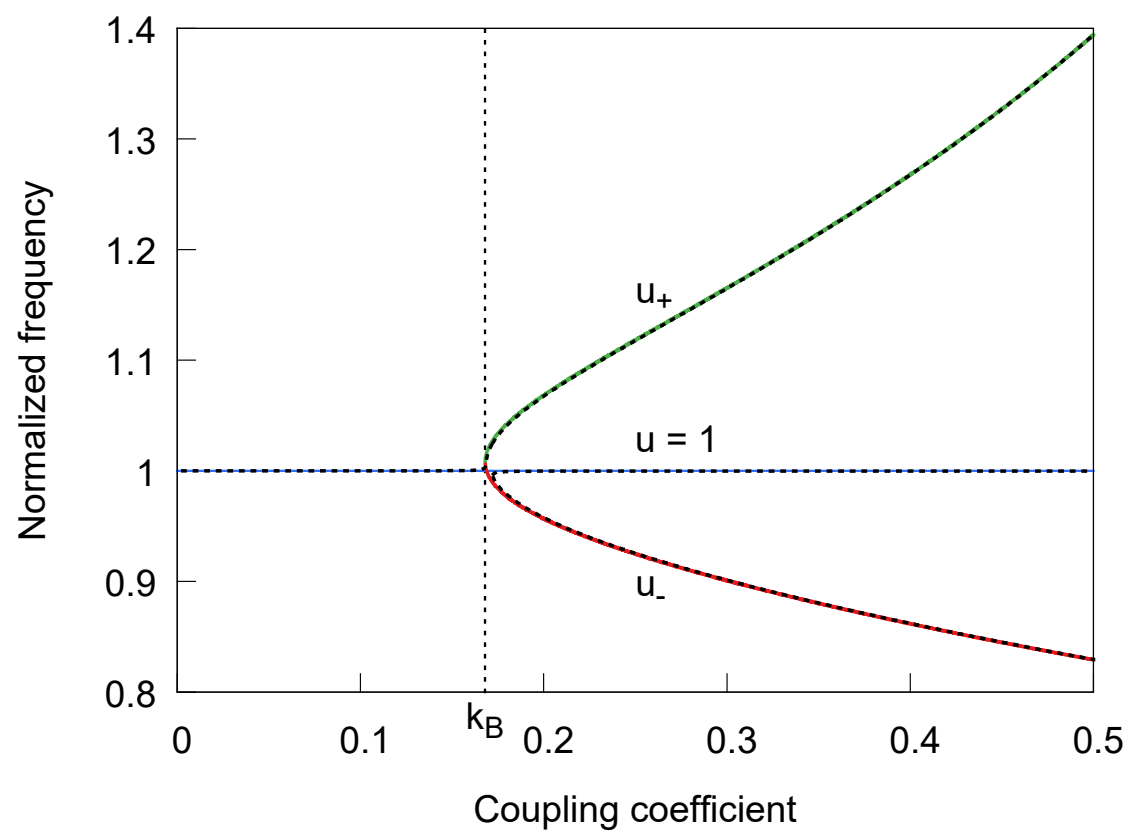

Figure 2. The main resonant frequency $u=1$ and the secondary frequencies $u_{ \pm}$as function of the coupling coefficient $k$ for the example configuration. Frequency bifurcation occurs for a coupling factor $k$ higher than the bifurcation coupling $k_{B}$. The solid lines indicate the results for the approximate admittance matrix $\boldsymbol{Y}_{a}$, the dashed lines for the exact admittance matrix $\boldsymbol{Y}$.

The shape of the bifurcation pattern depends on the values of the capacitor and inductor quality factors. If their values are such that from Equation (3) it results $\omega_{01}^{\prime}>\omega_{02}^{\prime}$, as in the present case, beyond the bifurcation point the main branch follows the upper resonant frequency, while the secondary branch 
includes the lower and the central resonant frequencies. For $\omega_{01}^{\prime}<\omega_{02}^{\prime}$ the roles of the lower and the upper resonances are interchanged. In the synchronous case $\left(\omega_{01}^{\prime}=\omega_{02}^{\prime}\right)$ the main branch follows the central resonant frequency $(u \approx 1)$ and a perfect pitchfork bifurcation is obtained from the exact model, too.

A practically perfect agreement between both models is observed, except in the immediate neighborhood of $k_{B}$, but even there, the perturbation is negligible small. We can conclude that if the quality factors are high, a prerequisite for most practical WPT systems, the analytical model sufficiently describes the system.

Figure 3 plots $G_{P}$ and $G_{T}$ as a function of the coupling factor $k$ for the approximated and exact model. No significant difference can be observed between the approximated and exact model. The higher the coupling $k$, the higher the power gain $G_{P}$ at main resonance. In the WPT literature, the power gain $G_{P}$ is often called the efficiency of the WPT link [31,32].



Figure 3. The gains $G_{P}$ and $G_{T}$ at the main resonant frequency $u=1$ and the secondary resonant frequencies $u_{ \pm}$(for $k>k_{B}$ ), as function of the coupling coefficient $k$ for the example configuration. The solid lines indicate the results for the approximate admittance matrix $Y_{a}$, the dashed line for the exact admittance matrix $Y$.

The transducer gain $G_{T}$ is a proportional measure for the power transfer to the load. For this example configuration, the transducer gain $G_{T}$ achieves a maximum at low coupling. The coupling coefficient $k_{c}$ that maximizes the power transfer can be found by maximizing Equation (27) as function of $k$. We find the optimal coupling coefficient $k_{c}$ of $6.5 \%$ (also called the critical coupling). For $k>k_{c}$, the output power decreases again.

Figure 3 also depicts $G_{P}$ and $G_{T}$ at the secondary resonant frequencies $u_{ \pm}$. Obviously, these values are only valid for a coupling higher than the bifurcation coupling $k_{B}$. When operating at $u_{ \pm}$, both $G_{P}$ and $G_{T}$ increase slightly for $u_{+}$, and decrease slightly for $u_{-}$. This indicates that the efficiency and power transfer remain fairly constant for varying coupling $\left(k>k_{B}\right)$. Notice the advantage of the coupling-independent mode for CPT applications: compared to the main resonant frequency, we obtain a higher power transfer to the load for $u_{ \pm}$(for $k>k_{B}$ ) at the expense of only a small reduction in efficiency (in particular for $u_{+}$). 


\section{Comparison to IPT}

CPT utilizes the electric field as means to transfer power wirelessly, whereas IPT uses the magnetic field. The equivalent network for IPT is shown in Figure 4. A voltage source $V_{S}$ with internal resistance $R_{S}$ supplies the transmitter. The inductances $L_{1}$ and $L_{2}$ are coupled by their mutual inductance $L_{M}$. The coupling factor $k_{I P T}$ is given by $k_{I P T}=L_{M} / \sqrt{L_{1} L_{2}}$.

Resonance capacitors $C_{1}$ and $C_{2}$ and resistances $R_{1}$ and $R_{2}$ are added in series to each circuit. Energy is transferred to the load $R_{L}$.

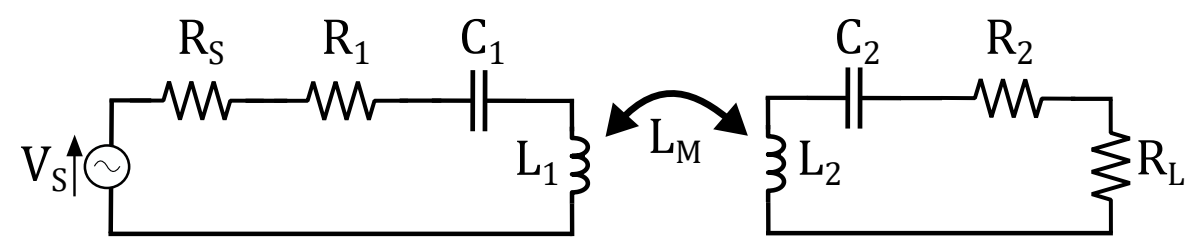

Figure 4. Equivalent circuit representation of a general inductive wireless power transfer (IPT) system.

We normalize according to Table 2 and define $r_{T x}=r_{1}+r_{S}$ and $r_{R x}=r_{2}+r_{L}$.

Table 2. Normalized quantities for IPT.

\begin{tabular}{ll}
\hline$r_{1}=R_{1} /\left(\omega_{0} L_{1}\right)$ & $r_{2}=R_{2} /\left(\omega_{0} L_{2}\right)$ \\
$r_{S}=R_{S} /\left(\omega_{0} L_{1}\right)$ & $r_{L}=R_{L} /\left(\omega_{0} L_{2}\right)$ \\
$r_{T x}=r_{1}+r_{S}$ & $r_{R x}=r_{2}+r_{L}$ \\
\hline
\end{tabular}

As was demonstrated in $[27,28]$, an analogous coupling-independent regime can be achieved for IPT. For this general IPT network, the power and transducer gain are given by:

$$
\begin{gathered}
G_{P, I P T}=\frac{k_{I P T}^{2} r_{L} u^{4}}{\left(r_{1}+k_{I P T}^{2} r_{R x}\right) u^{4}+r_{1}\left(r_{R x}^{2}-2\right) u^{2}+r_{1}} \\
G_{T, I P T}=\frac{4 k_{I P T}^{2} r_{S} r_{L} u^{6}}{D^{\prime}}
\end{gathered}
$$

with

$$
D^{\prime}=\left[\left(1-k_{I P T}^{2}\right) u^{4}-\left(2+r_{T x} r_{R x}\right) u^{2}+1\right]^{2}+\left[\left(r_{T x}+r_{R x}\right) u\left(1-u^{2}\right)\right]^{2}
$$

At the main resonance frequency $(u=1)$, the gains are given by:

$$
\begin{aligned}
& G_{P, I P T}(u=1)=\frac{k_{I P T}^{2} r_{L}}{r_{R x}\left(r_{1} r_{R x}+k_{I P T}^{2}\right)} \\
& G_{T, I P T}(u=1)=\frac{4 k_{I P T}^{2} r_{S} r_{L}}{\left(r_{T x} r_{R x}+k_{I P T}^{2}\right)^{2}}
\end{aligned}
$$

With the same methodology as for CPT, the secondary frequencies $u_{ \pm}$can be found:

$$
u_{ \pm, I P T}=\sqrt{\frac{2-r_{R x}^{2} \pm \sqrt{4 k_{I P T}^{2}+r_{R x}^{4}-4 r_{R x}^{2}}}{2\left(1-k_{I P T}^{2}\right)}}
$$

At $u_{ \pm}$, the power and transducer gain result in:

$$
G_{P, I P T}\left(u=u_{ \pm}\right)=\frac{r_{L}}{r_{1}+r_{R x}}
$$




$$
G_{T, I P T}\left(u=u_{ \pm}\right)=\frac{4 r_{S} r_{L}}{\left(r_{T x}+r_{R x}\right)^{2}}
$$

Both $G_{P, I P T}\left(u=u_{ \pm}\right)$and $G_{T, I P T}\left(u=u_{ \pm}\right)$are independent of the coupling between the transmitter and receiver coil.

The solid lines in Figure 5 show $G_{P, I P T}$ and $G_{T, I P T}$ as function of $k_{I P T}$ at main resonance, for the values $r_{1}=0.02, r_{2}=r_{S}=0.01$, and $r_{L}=0.15$. For $k_{I P T}>k_{B}$, the dashed lines plot $G_{P, I P T}$ and $G_{T, I P T}$ for the secondary resonances, which are coinciding for $u_{+}$and $u_{-}$. When operating at $u_{ \pm}$, both $G_{P}$ and $G_{T}$ remain constant. This indicates that the efficiency and power transfer remain constant for varying coupling $\left(k_{I P T}>k_{B}\right)$ : a perfect independent coupling is achieved for IPT [27,28]. In the previous section, we have shown that for $\mathrm{CPT}$, a perfect independent coupling is not attained. However, the change in $G_{P}$ and $G_{T}$ is almost negligible, resulting in a near independent coupling for practical WPT applications.

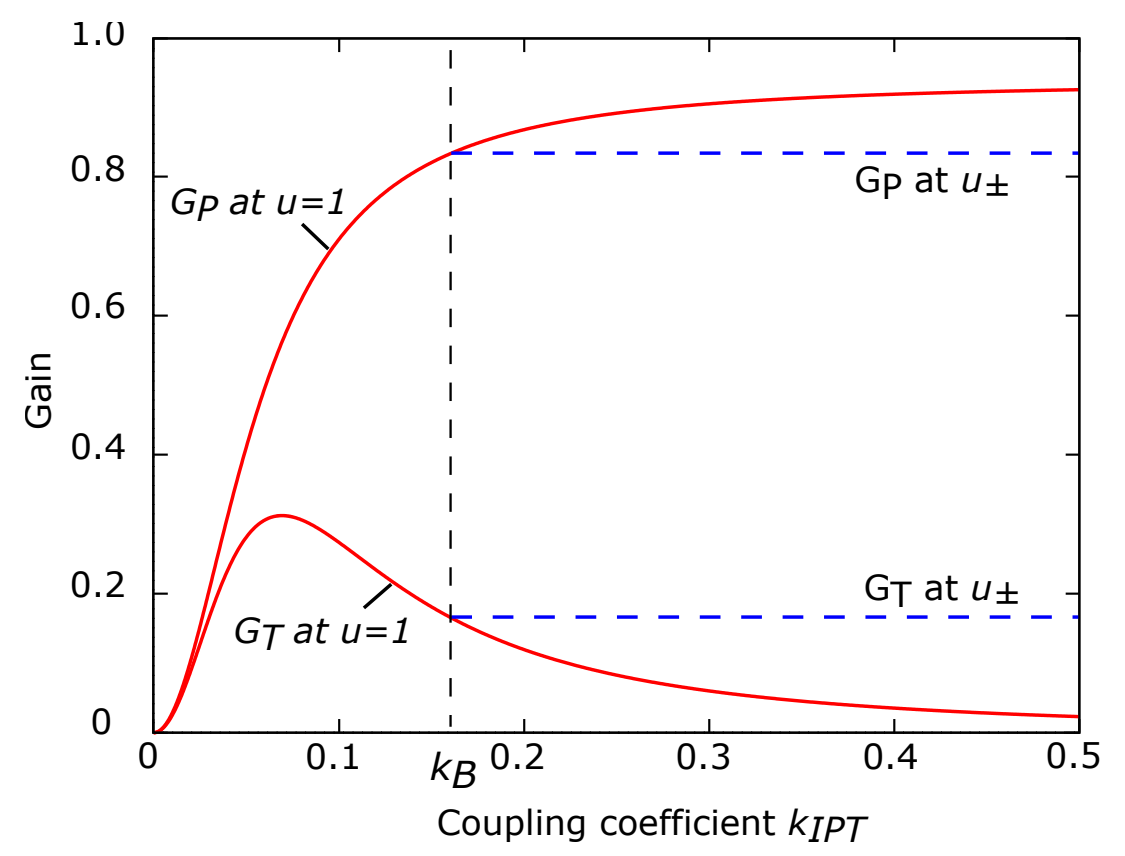

Figure 5. The continuous lines show the gains $G_{P, I P T}$ and $G_{T, I P T}$ at the main resonant frequency $u=1$ as function of the coupling coefficient $k_{I P T}$ for the example IPT configuration. The dashed lines indicate the values at the secondary resonant frequencies $u_{ \pm}$, valid for $k_{I P T}>k_{B}$.

\section{Conclusions}

We have calculated the analytical expressions for the power and transducer gain of a general CPT system. By determining the bifurcation conditions and frequencies, we have described the optimal solution for achieving a nearly coupling-independent CPT link. This solution allows for a purely resistive input admittance, leading to a nearly constant efficiency and power transfer for varying coupling. It allows for designs capable of obtaining a higher power transfer to the load for fluctuating coupling at the expense of a (small) reduction in efficiency. Measurements on an implemented CPT setup to confirm our analytical results are part of future research. Finally, we have illustrated an analogue configuration for IPT, where perfect coupling-independent power transfer is achievable.

Author Contributions: B.M., F.M. and M.M. performed the calculations and conducted the simulations. N.S. and A.C. assisted in supervising the calculations and simulations. B.M. wrote the manuscript. F.M., N.S., A.C. and M.M. commented on and revised the manuscript.

Funding: This research received no external funding.

Conflicts of Interest: The authors declare no conflict of interest. 


\section{Appendix A. Power Gain Expressions for a Two-Port as Function of the Admittance Parameters}

\section{Appendix A.1. Equivalent Circuit}

A two-port network can be characterized by its admittance matrix $Y$, with elements $y_{i j}=g_{i j}+j b_{i j}$ $(i, j=1,2)$. The following relations apply:

$$
I_{1}=y_{11} V_{1}+y_{12} V_{2} I_{2}=y_{21} V_{1}+y_{22} V_{2}
$$

At port 1 , we connect a sinusoidal current source $I_{S}$ (peak value) with internal admittance $Y_{S}=G_{S}+j B_{S}$ in parallel. At port 2, a load admittance $Y_{L}=G_{L}+j B_{L}$ is connected. This network can be represented by the equivalent circuit of Figure A1. From $Y_{L}=-I_{2} / V_{2}$ and Equation (A1), we find the input admittance $Y_{i n}=G_{i n}+j B_{i n}$ :

$$
Y_{i n}=y_{11}-\frac{y_{12} y_{21}}{y_{22}+Y_{L}}
$$

From $I_{1}=I_{S}-Y_{S} V_{1}$ and Equation (A1), we can determine the current at port 2:

$$
I_{2}=\left(y_{22}-\frac{y_{12} y_{21}}{y_{11}+Y_{S}}\right) V_{2}+\frac{y_{21}}{y_{11}+Y_{S}} I_{S}
$$

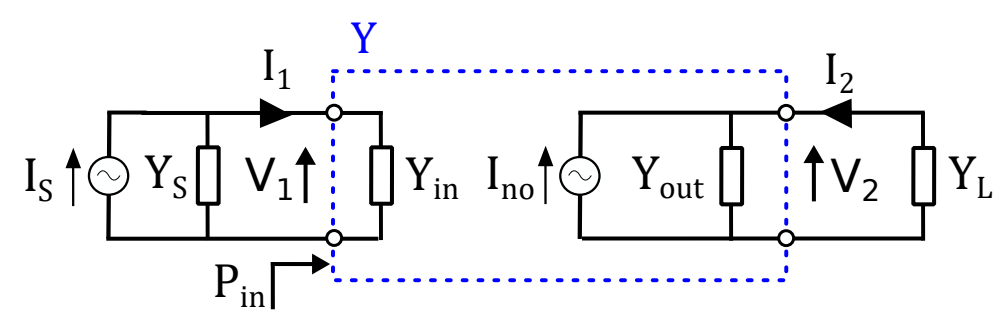

Figure A1. Equivalent circuit of a two-port network with load admittance $Y_{L}$ and current source $I_{S}$ with internal admittance $Y_{S}$. The input power $P_{\text {in }}$ is indicated.

We obtain the output admittance $Y_{\text {out }}=G_{\text {out }}+j B_{\text {out }}$ and the Norton equivalent current $I_{n o}$ :

$$
\begin{gathered}
Y_{\text {out }}=\left[\frac{I_{2}}{V_{2}}\right]_{I_{S}=0}=y_{22}-\frac{y_{12} y_{21}}{y_{11}+Y_{S}} \\
I_{n o}=\left[I_{2}\right]_{V_{2}=0}=\frac{y_{21}}{y_{11}+Y_{S}} I_{S}
\end{gathered}
$$

In the next subsections, we determine the power and transducer gain [31] for this general two-port network as function of its admittance parameters.

Appendix A.2. Power Gain

The input power $P_{\text {in }}$ of the two-port network is:

$$
P_{\text {in }}=\frac{1}{2} G_{i n}\left|V_{1}\right|^{2}
$$

The power $P_{L}$ dissipated by the load is:

$$
P_{L}=\frac{1}{2} G_{L}\left|V_{2}\right|^{2}
$$

The power gain $G_{P}$, often also called the efficiency of a WPT network, is defined as the ratio between the power $P_{L}$ and $P_{i n}$. We obtain: 


$$
G_{P}=\frac{P_{L}}{P_{i n}}=\frac{G_{L}}{G_{i n}}\left|\frac{V_{2}}{V_{1}}\right|^{2}
$$

With $Y_{L}=-I_{2} / V_{2}$ and Equation (A1), this results in the following expression for the power gain $G_{P}$ :

$$
G_{P}=\frac{G_{L}}{G_{i n}}\left|\frac{y_{21}}{y_{22}+Y_{L}}\right|^{2}
$$

Note that the power gain $G_{P}$ depends on the load admittance $Y_{L}$, but does not depend on the internal admittance $Y_{S}$.

Appendix A.3. Transducer Gain

The maximum input power $P_{A G}$, also called the available power of the generator, is given by [33]:

$$
P_{A G}=\frac{\left|I_{S}\right|^{2}}{8 G_{S}}
$$

The transducer gain $G_{T}$ is defined as the ratio between the power $P_{L}$ dissipated by the load and $P_{A G}$. For a fixed $P_{A G}$, maximizing $G_{T}$ corresponds to maximizing the amount of power transferred to the load.

With $V_{2}=I_{n o} /\left(Y_{\text {out }}+Y_{L}\right)$, Equations (A7) and (A10), we obtain the expression for the transducer gain $G_{T}$ :

$$
G_{T}=\frac{P_{L}}{P_{A G}}=\frac{4\left|y_{21}\right|^{2} G_{S} G_{L}}{\left|\left(y_{11}+Y_{S}\right)\left(y_{22}+Y_{L}\right)-y_{12} y_{21}\right|^{2}}
$$

Note that the transducer gain $G_{T}$ depends on both the load admittance $Y_{L}$ and the internal admittance $Y_{S}$.

\section{References}

1. Jawad, A.M.; Nordin, R.; Gharghan, S.K.; Jawad, H.M.; Ismail, M. Opportunities and challenges for near-field wireless power transfer: A review. Energies 2017, 10, 1022. [CrossRef]

2. Barman, S.D.; Reza, A.W.; Kumar, N.; Karim, M.E.; Munir, A.B. Wireless powering by magnetic resonant coupling: Recent trends in wireless power transfer system and its applications. Renew. Sustain. Energy Rev. 2015, 51, 1525-1552. [CrossRef]

3. Lu, X.; Wang, P.; Niyato, D.; Kim, D.I.; Han, Z. Wireless charging technologies: Fundamentals, standards, and network applications. IEEE Commun. Surv. Tutor. 2016, 18, 1413-1452. [CrossRef]

4. Mou, X.; Sun, H. Wireless power transfer: Survey and roadmap. In Proceedings of the 2015 IEEE 81st Vehicular Technology Conference (VTC Spring), Glasgow, UK, 11-14 May 2015; pp. 1-5.

5. Das, R.; Yoo, H. Wireless power transfer to a pacemaker by using metamaterials and yagi-uda antenna concept. In Proceedings of the 2015 International Workshop on Antenna Technology (iWAT), Seoul, Korea, 4-6 March 2015; pp. 353-354.

6. Kim, S. Wireless powering of miniaturized neurostimulator. In Proceedings of the 2017 IEEE International Symposium on Radio-Frequency Integration Technology (RFIT), Seoul, Korea, 30 August-1 September 2017; pp. 96-98.

7. $\mathrm{Xu}, \mathrm{Q} . ; \mathrm{Hu}, \mathrm{D}$.; Duan, B.; He, J. A fully implantable stimulator with wireless power and data transmission for experimental investigation of epidural spinal cord stimulation. IEEE Trans. Neural Syst. Rehabil. Eng. 2015, 23, 683-692. [CrossRef] [PubMed]

8. Zhang, J.; Zhu, C.; Chan, C. A wireless power charging method for automated guided vehicle. In Proceedings of the 2014 IEEE International Electric Vehicle Conference (IEVC), Florence, Italy, 17-19 December 2014; pp. 1-5.

9. Kawamura, A.; Ishioka, K.; Hirai, J. Wireless transmission of power and information through one high-frequency resonant AC link inverter for robot manipulator applications. IEEE Trans. Ind. Appl. 1996, 32, 503-508. [CrossRef] 
10. Cheng, Z.; Lei, Y.; Song, K.; Zhu, C. Design and loss analysis of loosely coupled transformer for an underwater high-power inductive power transfer system. IEEE Trans. Magn. 2015, 51, 1-10.

11. Covic, G.A.; Boys, J.T. Modern trends in inductive power transfer for transportation applications. IEEE J. Emerg. Sel. Top. Power Electron. 2013, 1, 28-41. [CrossRef]

12. Wu, H.H.; Gilchrist, A.; Sealy, K.; Bronson, D. A 90 percent efficient 5kW inductive charger for EVs. In Proceedings of the 2012 IEEE Energy Conversion Congress and Exposition (ECCE), Raleigh, NC, USA, 15-20 September 2012; pp. 275-282.

13. Shin, J.; Shin, S.; Kim, Y.; Ahn, S.; Lee, S.; Jung, G.; Jeon, S.J.; Cho, D.H. Design and implementation of shaped magnetic-resonance-based wireless power transfer system for roadway-powered moving electric vehicles. IEEE Trans. Ind. Electron. 2014, 61, 1179-1192. [CrossRef]

14. Choi, S.Y.; Gu, B.W.; Jeong, S.Y.; Rim, C.T. Advances in wireless power transfer systems for roadway-powered electric vehicles. IEEE J. Emerg. Sel. Top. Power Electron. 2015, 3, 18-36. [CrossRef]

15. Vamvakas, P.; Tsiropoulou, E.E.; Vomvas, M.; Papavassiliou, S. Adaptive power management in wireless powered communication networks: A user-centric approach. In Proceedings of the 2017 IEEE 38th Sarnoff Symposium, Newark, NJ, USA, 18-20 September 2017; pp. 1-6.

16. Xie, L.; Shi, Y.; Hou, Y.T.; Sherali, H.D. Making sensor networks immortal: An energy-renewal approach with wireless power transfer. IEEE/ACM Trans. Netw. 2012, 20, 1748-1761. [CrossRef]

17. Tsiropoulou, E.E.; Mitsis, G.; Papavassiliou, S. Interest-aware energy collection \& resource management in machine to machine communications. Ad Hoc Netw. 2018, 68, 48-57.

18. Ng, D.W.K.; Schober, R. Energy-efficient power allocation for M2M communications with energy harvesting transmitter. In Proceedings of the 2012 IEEE Globecom Workshops (GC Wkshps), Anaheim, CA, USA, 3-7 December 2012; pp. 1644-1649.

19. Kim, J.; Yang, S.Y.; Song, K.D.; Jones, S.; Elliott, J.R.; Choi, S.H. Microwave power transmission using a flexible rectenna for microwave-powered aerial vehicles. Smart Mater. Struct. 2006, 15, 1243. [CrossRef]

20. Minnaert, B.; Thoen, B.; Plets, D.; Joseph, W.; Stevens, N. Wireless energy transfer by means of inductive coupling for dairy cow health monitoring. Comput. Electron. Agric. 2018, 152, 101-108. [CrossRef]

21. Kim, T.H.; Yun, G.H.; Lee, W.Y.; Yook, J.G. Asymmetric Coil Structures for Highly Efficient Wireless Power Transfer Systems. IEEE Trans. Microw. Theory Tech. 2018, 66. [CrossRef]

22. Lu, F.; Zhang, H.; Mi, C. A Review on the Recent Development of Capacitive Wireless Power Transfer Technology. Energies 2017, 10, 1752. [CrossRef]

23. Kim, T.H.; Yoon, S.; Yook, J.G.; Yun, G.H.; Lee, W.Y. Evaluation of power transfer efficiency with ferrite sheets in WPT system. In Proceedings of the 2017 IEEE Wireless Power Transfer Conference (WPTC), Taipei, Taiwan, 10-12 May 2017; pp. 1-4.

24. Minnaert, B.; Stevens, N. Conjugate Image Theory Applied on Capacitive Wireless Power Transfer. Energies 2017, 10, 46. [CrossRef]

25. Minnaert, B.; Stevens, N. Single variable expressions for the efficiency of a reciprocal power transfer system. Int. J. Circ. Theory Appl. 2017, 45, 1418-1430. [CrossRef]

26. Halpern, M.E.; Ng, D.C. Optimal tuning of inductive wireless power links: Limits of performance. IEEE Trans. Circ. Syst. 2015, 62, 725-732. [CrossRef]

27. Costanzo, A.; Dionigi, M.; Mastri, F.; Mongiardo, M.; Monti, G.; Russer, J.A.; Russer, P. The basic cell operating regimes for wireless power transfer of electric vehicles. In Proceedings of the 2016 IEEE Wireless Power Transfer Conference, Aveiro, Portugal, 5-6 May 2016; pp. 1-4.

28. Mastri, F.; Costanzo, A.; Mongiardo, M. Coupling-independent wireless power transfer. IEEE Microw. Wirel. Compon. Lett. 2016, 26, 222-224. [CrossRef]

29. Zhang, W.; Wong, S.C.; Chi, K.T.; Chen, Q. Analysis and comparison of secondary series- and parallel-compensated inductive power transfer systems operating for optimal efficiency and load-independent voltage-transfer ratio. IEEE Trans. Power Electron. 2014, 29, 2979-2990. [CrossRef]

30. Huang, L.; Hu, A.P. Defining the mutual coupling of capacitive power transfer for wireless power transfer. Electron. Lett. 2015, 51, 1806-1807. [CrossRef]

31. Mastri, F.; Mongiardo, M.; Monti, G.; Dionigi, M.; Tarricone, L. Gain expressions for resonant inductive wireless power transfer links with one relay element. In Wireless Power Transfer; River Publishers: PW Delft, The Netherlands, 2017; pp. 1-15. 
32. Kiani, M.; Jow, U.M.; Ghovanloo, M. Design and optimization of a 3-coil inductive link for efficient wireless power transmission. IEEE Trans. Biomed. Circ. Syst. 2011, 5, 579-591. [CrossRef] [PubMed]

33. Montgomery, C.G.; Dicke, R.H.; Purcell, E.M. Principles of Microwave Circuits; Short Run Press Ltd.: British Exeter, UK, 1948.

(C) 2018 by the authors. Licensee MDPI, Basel, Switzerland. This article is an open access article distributed under the terms and conditions of the Creative Commons Attribution (CC BY) license (http://creativecommons.org/licenses/by/4.0/). 